\title{
Short-term healing response after implantation of the thin-strut, fast-releasing sirolimus-eluting biodegradable polymer-coated Alex Plus stent: optical coherence tomography study
}

\author{
Magdalena Dobrolińska ${ }^{1}$, Paweł Gąsior ${ }^{1}$, Tomasz Roleder ${ }^{2}$, Magda Roleder-Dylewska ${ }^{1}$, Grzegorz Smolka ${ }^{1}$, \\ Andrzej Ochała ${ }^{1}$, Elvin Kedhi ${ }^{1,3}$, Wojciech Wojakowski ${ }^{1}$ \\ ${ }^{1}$ Division of Cardiology and Structural Heart Diseases, Medical University of Silesia, Katowice, Poland \\ ${ }^{2}$ Regional Specialist Hospital, Research and Development Center, Wroclaw, Poland \\ ${ }^{3}$ AZ SintJan, Brugge, Belgium
}

Adv Interv Cardiol 2020; 16, 2 (60): 187-191 DOI: https://doi.org/10.5114/aic.2020.96062

\section{Introduction}

Technological improvements in drug-eluting stents (DES) have led to drastic reductions in restenosis and acute stent thrombosis rates. However, chronic inflammation and late and very late stent thrombosis, particularly with the first generation durable polymer DES (DPDES), prompted the development of new DES platforms that use biodegradable polymers. The advantage of biodegradable polymers relies on the resorbable property of these polymers, reducing in this way the polymer-induced chronic inflammation and related clinical events such as restenosis and stent thrombosis. Notwithstanding, despite the theoretical advantages of bioabsorbable polymer DES (BP-DES), there are limited data focused on its very early vascular healing process.

\section{Aim}

Therefore, we aimed to assess the 1-month vascular healing response following implantation of the bioabsorbable polymer-coated sirolimus-eluting stent Alex Plus (BP-SES, Alex Plus, Balton, Warsaw, Poland).

\section{Material and methods}

Study population

In this prospective, single-centre study, patients aged 18 years and older undergoing percutaneous coronary intervention $(\mathrm{PCl})$ for treatment of de novo coronary lesions with implantation of a bioresorbable polymer sirolimus-eluting stent (BP-SES), Alex Plus, for any clinical indication were enrolled. Major exclusive criteria were renal failure (glomerular filtration rate less than $45 \mathrm{ml} /$ $\mathrm{min} / 1.73 \mathrm{~m}^{2}$ ), allergy to contrast media or any of the stent components, hemodynamic compromise at presentation, pregnancy as well as participation in other clinical trials.

\section{Stent system description}

Alex Plus is composed of a laser-cut cobalt-chromium alloy stent platform with open-cell geometry and $71 \mu \mathrm{m}$ struts with a fully biodegradable circumferential coating (copolymer of poly-L-lactic and glycolic acid) and the anti-proliferative drug sirolimus. Experimental studies showed $95 \%$ release of the drug after 28 days and practically full polymer biodegradation after 8 weeks following Alex Plus implantation [1].

\section{Optical coherence tomography}

Optimal coherence tomography (OCT) using iLumien OPTIS Medical system (Abbott Vascular, Santa Clara, CA, USA) imaging was performed before and after stent implantation as well as at 1-month follow-up. The OCT registration included the entire stented segment as well as $5 \mathrm{~mm}$ proximally and distally to the stent. The OCT image was acquired using automated pullback triggered by the manual injection of contrast. The Review Workstation (Abbott, Santa Clara, CA, USA) was used for offline analysis of the treated segment. The region of interest was selected between the proximal and distal edges of the stent. The analysis was performed every $1 \mathrm{~mm}$ to measure lumen area (LA), lumen diameter (LD), endoluminal stent 
area (SA), minimal stent diameter, \% area stenosis (\%AS) and strut apposition to the vessel wall after implantation. Malapposition was defined as a visible space between the virtually drawn strut and the lumen contour or if the distance between the strut blooming and lumen counter was more than $71 \mu \mathrm{m}$. Furthermore, struts' coverage was also assessed at 1-month follow-up.

Based on OCT, the early coverage of each stent strut was classified using a previously published classification: definitely uncovered, uncovered fibrin, partially covered, covered protruding, covered embedded, malapposed (Figure 1). A definitely uncovered strut was defined as a strut not covered at any side. A partially covered strut was defined as a strut partially covered by tissue but only at one side. A covered protruding strut was defined as a strut covered by tissue on both sides but extending into the lumen. A covered embedded strut was defined as a strut covered by tissue or neointima and not extending into the lumen contour. An uncovered fibrin strut was defined as an uncovered strut covered with fibrin. A malapposed strut was present if the distance between the stent blooming artefact to the endoluminal surface was greater than the thickness of the strut equal of $71 \mu \mathrm{m}$. If the strut was covered with tissue, the thickness was measured as the distance between the blooming artefact and endoluminal surface [2].

The neointimal area was measured by subtracting as follows: endoluminal SA - (LA + malapposition area).

\section{Statistical analysis}

The Shapiro-Wilk test was used to analyse the continuous data distribution. Normally distributed values were presented as the mean \pm standard deviation. Non-normally distributed values were presented as the median with $25^{\text {th }}$ and $75^{\text {th }}$ percentile (interquartile range - IQR). Categorical variables were summarized using percentages and counts. Statistica (Statistica v. 13, Tibco Software Inc. Palo Alto, USA) was used for statistical analysis.

\section{Results}

\section{Study population}

Twelve patients underwent $\mathrm{PCl}$ with implantation of one Alex Plus per patient in de novo lesions in native coronary arteries. The mean patient's age was 71 years; $66.7 \%$ of them were males. Seventy-five percent of patients had chronic coronary syndrome (CCS), $25 \%$ of patients had acute coronary syndrome (ACS), and $17 \%$ had diabetes mellitus type 2 . All patients were treated with dual antiplatelet therapy (DAPT) during the entire duration of the study. One month of OCT imaging was performed in all patients.

\section{OCT analysis}

\section{Stent analysis}

The mean lesion length was $24.6 \pm 6.6 \mathrm{~mm}$. Before stent implantation, minimal LA and minimal LD were
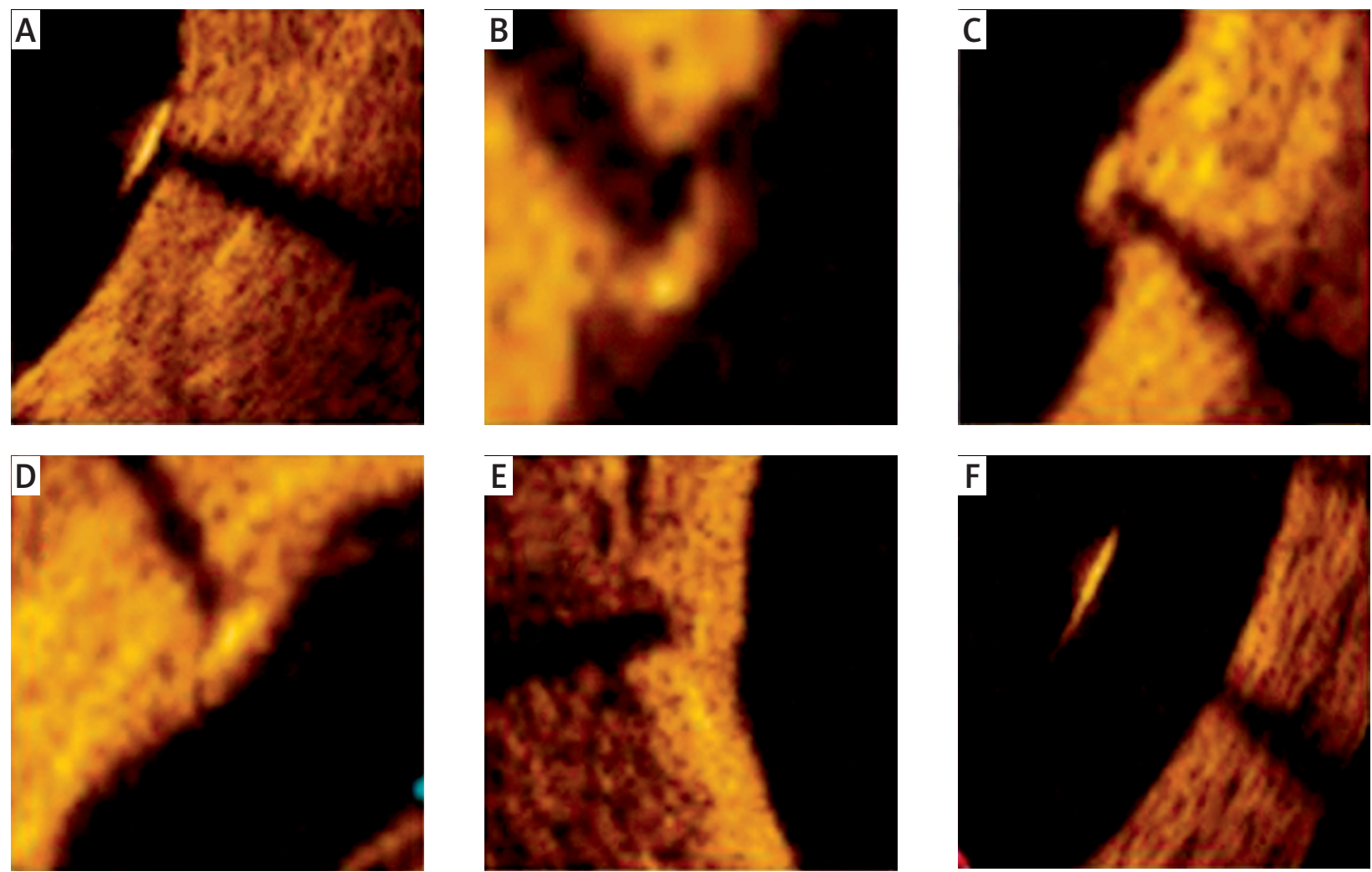

Figure 1. Optical coherence tomography-based stent strut coverage classification: definitely uncovered (A), uncovered fibrin (B), partially covered (C), covered protruding (D), covered embedded (E) and malapposed (F) 
respectively $2.3 \pm 2.2 \mathrm{~mm}^{2}$ and $1.51 \pm 0.6 \mathrm{~mm}$. The mean length of the implanted stents was $24.9 \pm 7.34 \mathrm{~mm}$. In a 1-month follow-up, the minimal LA and minimal LD were $5.98 \pm 2.73 \mathrm{~mm}^{2}$ and $2.58 \pm 0.58 \mathrm{~mm}$ respectively.

\section{Cross-section analysis}

In the follow-up, 2021 stent struts from 269 cross-sections were assessed (Figure 2). The mean neointimal thickness at covered struts was $12 \mu \mathrm{m}$ and the mean neointimal area was $0.62 \mathrm{~mm}^{2}$. The percent area stenosis at follow-up was $8.5 \pm 6.7 \%$. A total of 63 (3.12\%) stent struts were malapposed and mean malapposition area was $0.51 \pm 0.34 \mathrm{~mm}^{2}$. Furthermore, 67 (3.3\%) of all struts were defined as definitely uncovered, and in 9 stents, at least one strut was defined as definitely uncovered. Additionally, 1788 (88.5\%) struts had evidence of coverage (classified either as covered embedded or covered protruding). Stent analysis at 1-month follow-up is summarised in Table I.

\section{Discussion}

The major finding of this study is that the Alex Plus has a favourable coverage rate at 1 month.

First generation DP-DES significantly improved clinical outcomes compared to bare-metal stent (BMS); however, late and very late stent thrombosis following DPDES implantation was associated with polymer-induced chronic inflammation and hypersensitivity reactions [3]. Therefore, in the concept of using polymers which would fully degrade after completion of drug elution, BP-DES were designed to reduce those adverse effects of first generation DP-DES. The biodegradable polymer is designed to fully degrade from the strut surface after the elution of the anti-proliferative drug is completed. It was previously reported that the copolymer of poly-L-lactic and glycolic acid covering the assessed Alex Plus undergoes full bioresorption after 8 weeks [1]. Furthermore, the stent strut thickness was demonstrated to play an important role in the late stent thrombosis mechanism [4]. Additionally, previous studies showed that quicker and proper neointimal coverage might play a key role in shortening DAPT [5]. It was previously found that another cobalt-chromium BP-SES (BUMa Supreme, SINOMED, Tianjin, China) exhibited a higher rate of covered struts at 1-month follow-up as compared to cobalt-chromium durable polymer everolimus-eluting stent (DP-EES, XIENCE stent, Abbott Vascular, Santa Clara, CA, USA) [6]. In our study, the composition of the biodegradable coating and fast drug release kinetics, as well as low strut thickness of Alex Plus, resulted in a favourable healing profile expressed by the relatively high rate $(88.5 \%)$ of covered struts (embedded covered and protruding covered struts). The results of our study, which showed $11.5 \%$ of struts without evidence of complete coverage,
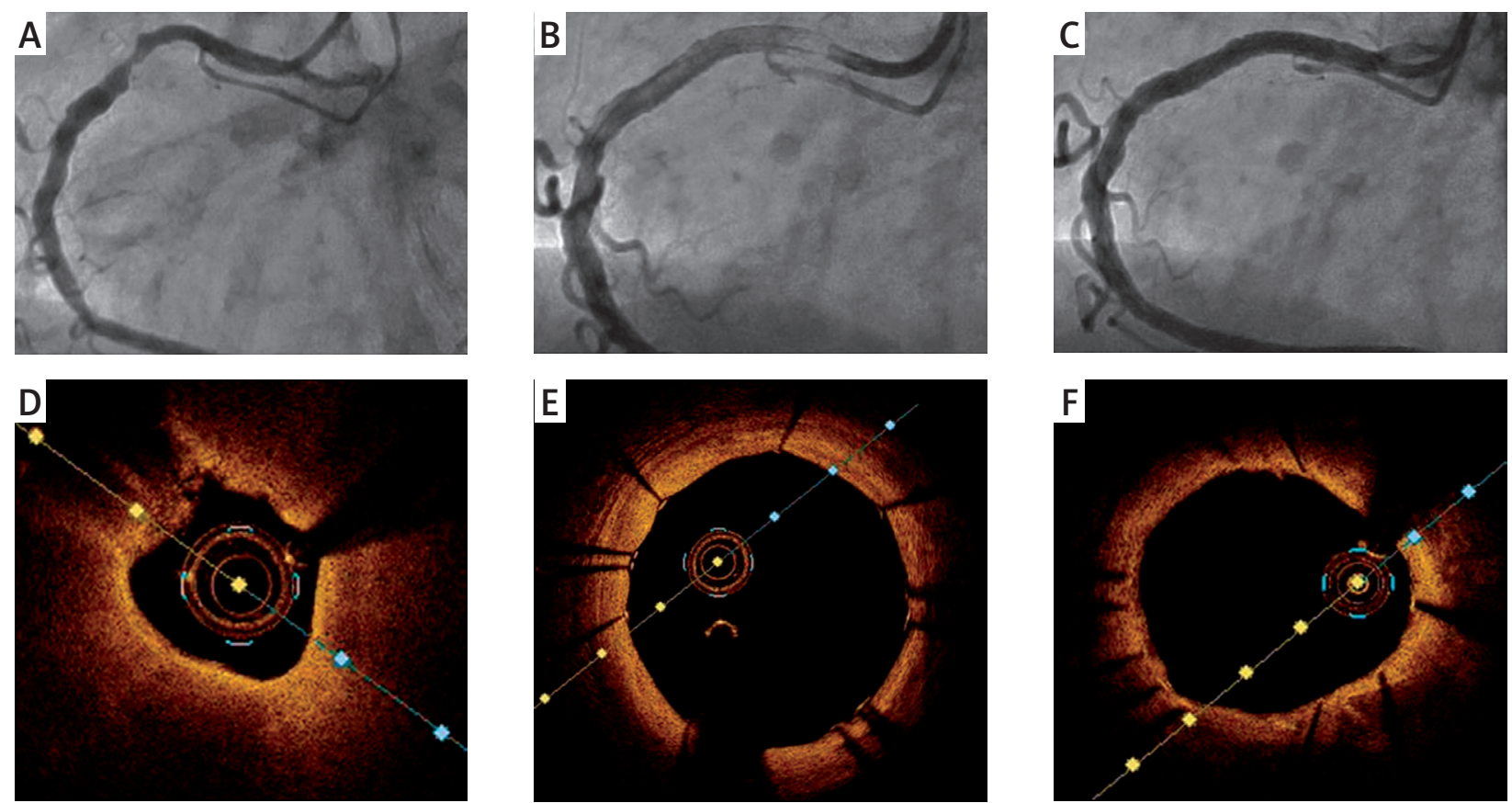

Figure 2. Representative optical computed tomography (OCT) imaging before and after Alex Plus implantation as well as at 1-month follow-up. A - Baseline angiography of a patient with stenosis of the right coronary artery (RCA). B - Implantation of Alex Plus stent. C - Angiography at the 1-month follow-up. D - OCT cross-sectional image before Alex Plus implantation. E - OCT cross-sectional image after Alex Plus implantation. F - OCT cross-sectional image at the 1-month follow-up 
Table I. OCT analysis at baseline and 1-month follow-up

\begin{tabular}{|c|c|}
\hline Quantitative findings & Result \\
\hline \multicolumn{2}{|l|}{ Stent implantation: } \\
\hline Left anterior descending artery & $1(8 \%)$ \\
\hline Left circumflex artery & $8(67 \%)$ \\
\hline Right coronary artery & $3(25 \%)$ \\
\hline \multicolumn{2}{|l|}{ Lesion: } \\
\hline Lesion length [mm] & $24.6 \pm 6.6$ \\
\hline Minimal lumen area $\left[\mathrm{mm}^{2}\right]$ & $2.3 \pm 2.2$ \\
\hline Minimal lumen diameter [mm] & $1.51 \pm 0.6$ \\
\hline$\%$ Area stenosis & $60.0 \pm 22.7$ \\
\hline Calcium presence & $12(100 \%)$ \\
\hline TCFA presence & $7(63 \%)$ \\
\hline \multicolumn{2}{|l|}{ 1-month follow-up: } \\
\hline ROI length [mm] & $24.9 \pm 7.34$ \\
\hline Proximal reference lumen area & $8.41 \pm 3.63$ \\
\hline Distal reference lumen area & $6.5 \pm 3.76$ \\
\hline Minimal lumen area & $5.98 \pm 2.73$ \\
\hline Minimal lumen diameter & $2.58 \pm 0.58$ \\
\hline Minimal stent area & $6.41 \pm 2.31$ \\
\hline Minimal stent diameter & $2.55 \pm 0.45$ \\
\hline Neointimal area & $0.62(0.22-0.85)$ \\
\hline Neointimal thickness & $0.12(0.1-0.17)$ \\
\hline Malapposition area & $0.51 \pm 0.34$ \\
\hline$\%$ Area stenosis & $8.5 \pm 6.7$ \\
\hline \multicolumn{2}{|l|}{ Strut level analysis: } \\
\hline \multicolumn{2}{|l|}{ Strut coverage } \\
\hline Definitely uncovered (A) & $67(3.3 \%)$ \\
\hline Uncovered fibrin (B) & $26(1.3 \%)$ \\
\hline Partially covered (C) & $140(6.9 \%)$ \\
\hline Covered protruding (D) & $384(19.0 \%)$ \\
\hline Covered embedded (E) & 1404 (69.5\%) \\
\hline Total uncovered $(A+B+C)$ & $233(11.5 \%)$ \\
\hline Malapposed struts (F) & $63(3.12 \%)$ \\
\hline Total number of covered struts $(D+E)$ & $1788(88.5 \%)$ \\
\hline Total number of struts & $2021(100 \%)$ \\
\hline
\end{tabular}

are favourable as compared to the previously published 1-month coverage for another cobalt-chromium BP-SES (Orsiro DES, Biotronik AG, Bulach, Switzerland) which demonstrated $19.6 \%$ uncovered struts analysed in patients with ST elevation myocardial infarction [7]. However, it needs to be stressed that the higher rate of the uncovered struts might be attributed to the exclusively ST-elevation myocardial infarction (STEMI) population.

Despite the reduction of the chronic inflammation process, late stent thrombosis still occurs after implantation of BP-DES. Pathological studies showed that late stent thrombosis might also be a result of uncovered and malapposed struts' presence [8]. As compared to DP-ZES, we observed a numerically lower percentage of malapposed and uncovered struts (BP-SES $5.7 \%$ vs. DP-ZES $3.12 \%$ and BP-SES $12.3 \%$ vs. DP-ZES $4.6 \%$ respectively) [9]. Additionally, our results are comparable to EGOBIOFREEDOM results of 1-month follow-up after implantation of thick-strut $(112 \mu \mathrm{m})$ polymer-free DES $(85.8 \%$ of struts defined as covered) [10]. Furthermore, results of a large randomized study demonstrated that among patients at high bleeding risk, the 1-month DAPT after DP-ZES implantation therapy was non-inferior to a polymer-free DES with regard to death from cardiac causes, myocardial infarction or stent thrombosis [11, 12]. In summary, our study demonstrated comparable strut coverage and a favourable healing profile when compared to the previous study regarding 1-month healing response after DP-ZES implantation [9]. It may suggest that the evaluated Alex Plus might be considered as a potential candidate for short DAPT therapy in the future. However, a large randomized clinical trial is needed for verification of the results of this study.

There are several study limitations that have to be mentioned. First, the small population of the study might bias the final results. The study assessed only the sirolimus stent platform and did not provide data for a control group. Moreover, OCT does not distinguish healthy from abnormal tissue, which may overestimate the final assessment of the stent healing process.

\section{Conclusions}

The 1-month OCT follow-up of thin-strut Alex Plus showed a high proportion of covered struts, suggesting a favourable healing profile.

\section{Acknowledgments}

Funded by Medical University of Silesia statutory funds (PCN-640-2-1-125/20).

\section{Conflict of interest}

The authors declare no conflict of interest.

\section{References}

1. Orlik B, Buszman PP, Krauze A, et al. A nuclear magnetic resonance spectroscopy as a method for evaluation of in vivo poly-l-lactide biodegradation kinetics from stent-polymer matri- 
ces: an experimental study utilizing porcine model of in-stent restenosis. J Cardiovasc Pharmacol Ther 2016; 21: 93-6.

2. Vesga B, Hernandez H, Moncada M, et al. Three-month evaluation of strut healing using a novel optical coherence tomography analytical method following bioresorbable polymer everolimus-eluting stent implantation in humans: the TIMELESS study. Coron Artery Dis 2017; 28: 126-34.

3. Stettler C, Wandel S, Allemann S, et al. Outcomes associated with drug-eluting and bare-metal stents: a collaborative network meta-analysis. Lancet 2007; 370: 937-48.

4. Lupi A, Gabrio Secco G, Rognoni A, et al. Meta-analysis of bioabsorbable versus durable polymer drug-eluting stents in 20,005 patients with coronary artery disease: an update. Catheter Cardiovasc Interv 2014; 83: E193-206.

5. Finn AV, Joner M, Nakazawa G, et al. Pathological correlates of late drug-eluting stent thrombosis: strut coverage as a marker of endothelialization. Circulation 2007; 115: 2435-41.

6. Asano T, Jin Q, Katagiri Y, et al. A randomised comparison of healing response between the BuMA Supreme stent and the XIENCE stent at one-month and two-month follow-up: PIONEER-II OCT randomised controlled trial. Eurolntervention 2018; 14: e1306-15.

7. Secco GG, Mattesini A, Fattori R, et al. Time-related changes in neointimal tissue coverage of a novel sirolimus eluting stent: serial observations with optical coherence tomography. Cardiovasc Revasc Med 2016; 17: 38-43.

8. Cook S, Wenaweser P, Togni M, et al. Incomplete stent apposition and very late stent thrombosis after drug-eluting stent implantation. Circulation 2007; 115: 2426-34.

9. Roleder T, Kedhi E, Berta B, et al. Short-term stent coverage of second-generation zotarolimus-eluting durable polymer stents: Onyx one-month optical coherence tomography study. Adv Interv Cardiol 2019; 15: 143-50.

10. Lee SWL, Tam FCC, Chan KKW, et al. Establishment of healing profile and neointimal transformation in the new polymer-free biolimus A9-coated coronary stent by longitudinal sequential optical coherence tomography assessments: the EGOBIOFREEDOM study. Eurolntervention 2018; 14: 780-8.

11. Urban P, Meredith IT, Abizaid A, et al. Polymer-free drug-coated coronary stents in patients at high bleeding risk. N Engl J Med 2015; 373: 2038-7.

12. Windecker S, Latib A, Kedhi E, et al. Polymer-based or polymer-free stents in patients at high bleeding risk. N Engl J Med 2020; 382: 1208-18. 